\title{
Labyrinthe
}

$30 \mid 2008$ (2)

Ecologie $=X$

\section{Écologiste, encore un effort pour devenir antihumanistes...}

Écologie, humanisme et capitalisme

Frédéric Neyrat

\section{(2) OpenEdition}

\section{Journals}

Édition électronique

URL : https://journals.openedition.org/labyrinthe/3776

DOI : 10.4000/labyrinthe.3776

ISSN : 1950-6031

Éditeur

Hermann

Édition imprimée

Date de publication : 10 juin 2008

Pagination : 101-111

ISBN : 978-2-9526131-7-0

Référence électronique

Frédéric Neyrat, "Écologiste, encore un effort pour devenir antihumanistes... », Labyrinthe [En ligne] 30 | 2008 (2), mis en ligne le 27 mai 2008, consulté le 14 janvier 2022. URL : http://

journals.openedition.org/labyrinthe/3776 ; DOI : https://doi.org/10.4000/labyrinthe.3776

Propriété intellectuelle 


\title{
ÉCOLOGISTES, ENCORE UN EFFORT POUR DEVENIR ANTIHUMANISTES... Écologie, humanisme et capitalisme
}

\author{
Frédéric NEYRAT \\ fredericneyrat@free.fr
}

Dans l'entreprise de déqualification systématique que de nombreux essayistes, philosophes, romanciers, hommes de pouvoir et journalistes appliquent quotidiennement à la pensée de l'écologie, l'attaque la plus prisée consiste à dire que l'écologie, dans son principe, quand on la laisse s'exprimer jusqu'à son terme, est «antihumaniste». Cette déqualification est à ce point assurée qu'elle occupe non seulement les débats, mais induit chez de nombreux écologistes la préparation d'un argumentaire préventif susceptible de parer à l'infâmante épithète. L'attaque comme la défense sont cependant hautement problématiques, en ceci que l'une et l'autre récusent le tranchant de la pensée écologiste.

De part et d'autre, «antihumanisme» signifie: «haine de l'homme», autrement dit «misanthropie». On dira donc, d'un côté, que les écologistes au fond n'aiment pas les hommes; de l'autre, qu'on les aime. D'un côté que les écologistes en veulent à l'humanité de l'homme au point de vouloir - secrètement ou d'une façon plus assumée - l'immerger, âme et libre-arbitre, dans la Nature ou quelque Grand-Tout vivant; de l'autre qu'en combattant pour la Nature, on combat en définitive pour l'Homme-et-son-environnement. Notre objectif dans cet article consistera à contester les fondements de cette critique comme de cette défense. Pour ce faire, nous irons au cœur du problème, en rapportant les enjeux de la fameuse controverse qui a opposé en 1989 Murray Bookchin, l'un des représentants les plus importants de l'écologie sociale, figure de proue de la «nouvelle gauche» écologiste américaine, et Dave Foreman, l'un des fondateurs du mouvement américain Earth First! accusé par Bookchin d' "antihumanisme». Or, cette controverse repose selon nous sur une explicitation insuffisante de l'humanisme: l'humanisme n'est pas un sentiment pour l'homme, mais une fiction rétroactive qui s'est installée au moment de l'essor du capitalisme. 
Ce que nous appelons aujourd'hui du terme d'humanisme n'est en vérité qu'un mode de production de l'humanité compatible avec le capitalisme.

L'écologie pensée jusqu'au bout, c'est-à-dire la "pensée écologisée» (Edgar Morin) et l'écologie politisée, est ce qui peut nous permettre de comprendre le rapport entre humanisme et capitalisme: l'humanisme du capital, c'est ni plus ni moins que le nom d'une production singulière de l'être humain comme être d'exception'. Et l'écologie politique est ce qui repère les effets désastreux d'une telle production. En ce sens, nous voulons montrer qu'une politique «écologisée» comme une écologie bien pensée ne peuvent conduire qu'à un antihumanisme vigoureux, n'ayant rien à voir avec quelque ressentiment envers l'homme.

\section{La controverse Bookchin-Foreman}

Dès 1987, Bookchin avait initié une attaque très virulente contre le courant de la deep ecology, qu'il avait accusée d' "antihumanisme». Voici les propos de Foreman, cités par Bookchin en 1989: «Il est temps pour cette société guerrière de disparaître de la terre dans un raz-demarée destructeur qui formera des anticorps contre la vérole humaine qui est en train de ravager cette belle planète ${ }^{2}$." Il s'agirait donc, pour les deep ecologists, de dépeupler la terre, de «laisser faire la nature " et de louer l'occurrence des désastres naturels et des génocides passifs. Il est vrai que Foreman, et c'est cela qui avait mis le feu aux poudres de l'attaque bookchinienne, avait tenu des propos pour le moins inquiétants sur l'inutilité de l'aide humanitaire en direction d'enfants mourant de faim au Sahel...

Bookchin est pourtant d'accord avec les deep ecologists pour identifier les désastres écologiques et la «nécessité d'apprendre à vivre en harmonie avec la planète, avec le reste de la biosphère», d'accord aussi sur la nécessité de «trouver une solution rationnelle au problème de la population» $[Q$, p. 40$]$. Mais, soutient-il, il ne faut pas se tromper d'en-

\footnotetext{
1. Nous découvrons et lisons le livre de Jean-Marie Schaeffer, La Fin de l'exception humaine, Paris, Gallimard, 2007. II nous faudra y revenir ultérieurement. Mais, d'ores et déjà, on peut soutenir que l'exception humaine n'a jamais été aussi forte, aussi globalisée qu'aujourd hui.

2. Murray Bookchin et Dave Foreman, Quelle écologie radicale?, Lyon, Atelier de création libertaire/Silence, 1994, p. 31 [ouvrage dorénavant noté $Q$ ].
} 
nemi: les causes du désastre, ce n'est pas l'humanité en général, $c$ 'est le capitalisme, le rapport de production capitaliste, la cause est socioéconomique. Ce qu'il faut donc commencer par faire, c'est étudier les pouvoirs et les institutions telles qu'elles sont, car ce sont ces dernières qui déterminent notre rapport à la nature: «Le concept même de domination de la nature provient de la domination de l'homme par l'homme " $[Q$, p. 99]. D'accord, nous dit-il, il y a bien une maladie qui nous accable, le capitalisme est bien le «cancer de la biosphère» $[Q, \mathrm{p} .65]$, mais la maladie ce n'est pas l'homme, c'est cette société. Le mal est ancien: «La société est empoisonnée. Elle a été empoisonnée il y a des milliers d'années, avant l'âge du Bronze. Elle a été faussée par les règles des anciens, par le patriarcat, par les guerriers, par des hiérarchies de toutes sortes qui ont conduit à la situation actuelle d'un monde menacé par des États-nations en compétition, possédant l'arme nucléaire et par un système capitaliste de grandes sociétés incroyablement destructeur à l'Ouest, et un système capitaliste d'État bureaucratique, également destructeur sur le plan écologique bien que périclitant, à l'Est " $[Q$, p. 44]. Par rapport à ce mal radical, dépeupler serait largement insuffisant: il ne s'agit pas de dépeupler, mais de transformer.

Foreman répond en montrant tout d'abord que le capitalisme ne se réduit pas à la domination d'une classe sur une autre - et refuse ainsi à la gauche sociale d'exempter les «travailleurs» de la «destruction du monde naturel » $[Q$, p. 59]. Deuxièmement, la volonté consistant à préserver une nature vierge, qui justifie le slogan des eco-warriors - «Pas de compromis dans la défense de la Terre-Mère» - ne consiste nullement à couper celle-ci de la société, mais à limiter le phagocytage de la nature par la société: il s'agit, pourrait-on dire, de rappeler l'existence de la nature face à son déni, c'est-à-dire au moment de sa destruction. Et de rappeler l'existence de la nature jusque dans ce qui la nie: "Quand les gens me parlent de destruction de propriété, de bulldozers démoniaques qui détruisent, tout ce que je peux dire, c'est qu'un bulldozer est fait de minerai de fer. C'est une partie de la terre. Un bulldozer, c'est la Terre métamorphosée en monstre qui s'autodétruit. En le sabotant, vous libérez le dharma nature du bulldozer, et vous le ramenez à la Terre" $[Q$, p. 53]. Troisièmement, Foreman précise que cette wilderness est nécessaire à l'autoréalisation de l'humanité comme à la diversification créatrice des espèces végétales et animales: comme le soutient Arne Naess dans Ecology, Community and Lifestyle, la préser- 
vation de la nature n'a rien de réactionnaire, c'est une pratique et un désir éminemment dynamiques ${ }^{3}$. Enfin, Foreman rappelle que le capitalisme est sous condition d'un humanisme «où les êtres humains sont la mesure de toute valeur» $[Q$, p. 53], et déclare ici son accord avec l'ouvrage de David Ehrenfeld de 1978: The Arrogance of Humanism.

Et c'est bien autour de ce dernier point que tourne l'opposition de Bookchin à Foreman. Pour Bookchin, ce rejet de l'humanisme ferait de l'écologie profonde le miroir inversé de ce qu'elle critique: «anthropocentrisme antinature» et "aversion misanthrope envers l'espèce humaine» $[Q$, p. 43$]$ sont au fond d'accord pour penser l'être humain comme un extra-terrestre, comme un être se voulant contre-nature. Ou bien l'on fait de cette qualité extra-terrestre un privilège, ou bien le motif d'une répudiation. Rejet des Lumières, "esprit de clocher», «modes antirationnelles» au crible desquelles transitent les lois de la nature, «biocentrisme» voulant réduire l'homme à quelque chose de purement et simplement biologique, tout cela mène à l' «écofascisme" sur fond d'un slogan minimal: «À bas les êtres humains». "N'autorisons pas que la célébration de la nature comme une fin en soi ne dégénère en un antihumanisme misanthrope», déclare Bookchin, mais exigeons un «humanisme écologiste étendu» $[Q, p$. 67]. Ce qui veut dire: socialiser l'écologie, écologiser le social. Contre le biocentrisme et l'anthropocentrisme, un "naturalisme dialectique», une «éthique de la complémentarité» pouvant s'appliquer aussi bien à la nature "première" qu'à la «nature seconde», ce «réseau culturel» qui s'ajoute aux «corps de primates» des humains. Désormais, «le projet de libération de l'homme est devenu un projet écologiste, tout comme à l'inverse, le projet de protection de la terre est devenu un projet social », et l'écologie sociale « rassemble ces deux projets» $[Q$, p. 126-127].

Beau projet de réconciliation des natures vers ce que Bookchin nomme une "nature libre», belle dialectique qui n'oublie rien, évitant tout excès. Le problème, c'est que la situation actuelle infirme la possibilité de cette dialectique. Pour qu'il y ait une Aufhebung de la nature "première» et de la nature «seconde» en une «nature libre», encore faudrait-il que la première ait encore la force de s'autodépasser, et ne se perde pas définitivement dans le néant du passage dialectique en son

3. Sur ce point, voir Michael Zimmerman, Contesting Earth's Future, Berkeley, University of California Press, 1994, p. 165-166. 
contraire. Ce que Bookchin voit sans pourtant en tirer toutes les conséquences, c'est l'asymétrie destructrice des natures; ce que Foreman voit mieux que lui, c'est le fait que l'asymétrie est telle qu'elle interdit telle quelle, immédiatement, la belle réconciliation. Il faut donc parer à l'usage trop intrusif de l'homme ${ }^{4}$. Pour cette raison, Bookchin se trompe lorsqu'il reproche aux deep ecologists leur propre clivage nature/culture, car ce clivage est d'abord un effet de civilisation, et les deep ecologists ne font que proposer un changement profond de civilisation, qui passe forcément par la valorisation de ce qui a été dévalorisé: «Le bien-être humain, dit Foreman, est vitalement important pour moi, mais il n'est pas l'ultime valeur éthique. Je suis d'accord avec Aldo Leopold qu'en fin de compte une chose est bonne lorsqu'elle tend à mettre en valeur l'intégrité, la stabilité et la beauté de la communauté biotique» $[Q$, p. 114]. Et, pour Foreman, il n'est pas du tout certain que la modification des relations sociales entre les hommes changera quoi que ce soit au sort de la "communauté biotique», l'égalité entre les hommes ne changera rien à l'anthropocentrisme, rien à la domination de la nature comme le dit Warwick Fox ${ }^{5}$. Car l'égalité entre les hommes maintient le privilège humaniste.

En définitive, comment faut-il se rapporter à cette controverse, quelle leçon en tirer? Ce qu'il faut comprendre, c'est la raison pour laquelle ces deux discours glissent l'un sur l'autre, et ne parviennent pas à se croiser comme il faut. Leur surdité mutuelle (qu'il faudrait faire résonner avec certaines interrogations actuelles de la gauche française, lorsqu'elle tente de faire dialoguer un écologiste avec un syndicaliste $^{6}$ ) tient à leur incapacité à mettre en rapport la question de la place de l'homme avec celle du capitalisme. Et ce rapport implique une compréhension de l'humanisme à laquelle n'accèdent ni Foreman ni Bookchin, qui réduisent l'humanisme à un sentiment et une appréciation axiologique. Si Foreman a parfaitement compris qu'on ne changera rien aux désastres écologiques sans remettre en cause la place de I'homme, de tout homme et quelle que soit sa classe ou son groupe d'appartenance socio-économique, il ne comprend pas en quoi cette place est produite par un contexte idéopratique, écotechnique, et en

4. Ibidem, p. 153.

5. Ibidem. p. 178.

6. Jean Gadrey, «L'écologiste et le syndicaliste n, http://www.alternatives-economiques.fr/blogs/gadrey/ [consulté le 15 mai 2008]. 
l'occurrence capitaliste; si Bookchin comprend parfaitement la fonction cruciale du capitalisme, il ne voit pas en quoi celui-ci poursuit un schème de production transhistorique qui situe l'être humain - tout être humain - en exception. L'un croit qu'il suffit de changer la place de l'homme; l'autre, le système économique - aucun n'interroge la façon dont le capitalisme doit être compris ontologiquement, comme mode de production de l'humanité, comme anthropotechnique singulière. Aucun des deux ne comprend le phénomène de la mondialisation de l'humanisme, qui n'est pas qu'une histoire de peuplement comme le soutient Foreman, et qui ne serait qu'accentué par la dialectique bookchinienne: la mondialisation de l'humanisme, c'est la production d'un monde à l'usage d'un être en exception vis-à-vis de ce monde. Le capitalisme est l'instrument le plus adapté pour réaliser un tel projet. Autrement dit, le capitalisme est un humanisme.

\section{Le capitalisme est un humanisme}

Une telle formule doit être éclairée, car elle est pour le moins bizarre: en quoi les ravages du capitalisme pourraient-ils être interprétés comme le signe d'une promotion de la dignité de l'homme? Ce que nous voulons dire, c'est que la formation d'une Valeur humaniste coupée de tout rapport avec le non-humain conduit à ne pouvoir donner qu'un prix dérisoire à tout ce qui constitue le monde. Cela, nous ne pouvons le dire qu'en articulant la pensée écologiste, une interprétation de l'humanisme et une compréhension ontologique du capitalisme. Cette articulation doit s'effectuer autour de la situation d'exception qui est faite à l'humanité, car c'est bien autour des effets de la production capitaliste de l'exception humaniste que s'enroule en définitive la discussion entre Bookchin et Foreman.

Si l'écologie peut être considérée comme une pensée et pas seulement comme une pratique, une théorie, une science ou un argument publicitaire, c'est qu'elle tranche sur les savoirs établis, c'est qu'elle exige la remise en cause d'un paradigme qui a guidé - qui a, au sens le plus profond du terme, schématisé - l'avènement de la civilisation globale contemporaine. Ce paradigme a consisté à attribuer une place exceptionnelle à l'être humain. Mais il est diverses manières de s'excepter du monde, et l'on ne doit jamais oublier cette diversité: 
1/ en se situant au centre de celui-ci, non pas en dehors mais au centre (quitte à chercher le meilleur moyen pour en sortir), et c'est ce que l'époque grecque pourrait symboliser; $2 /$ on peut aussi, comme pour l'époque chrétienne, et en général pour tout système monothéologique, situer l'homme comme le sommet de la Création (quand bien même ce sommet est une limite indépassable, qu'un gouffre sépare de son AuDelà) ; 3/ on peut enfin envisager l'homme comme extérieur au monde: comme l'écrivent Catherine Larrère et Raphaël Larrère, Descartes pose l'homme hors du monde, «en position d'expérimentation et de maîtrise $^{7}$ » (quitte à chercher par tous les moyens à être, enfin, au-monde ou dans-le-monde-avec-les-autres). Pour Catherine et Raphaël Larrère, nous serions en passe de voir aujourd'hui l'homme «réinscrit» dans la nature, mais «sans position privilégiée» $[D, \mathrm{p} .18]$. Refusant de participer à la «dénonciation de la deep ecology ou de l'écocentrisme», ils cherchent une "position écocentrée» capable d'éviter la «menace antihumaniste» $[D$, p. 301-302], et se demandent: «Tout naturalisme est-il nécessairement antihumaniste?» Non, nous disent-ils, on peut concilier les deux car l'homme «fait partie de la nature», et il n'y a pas à choisir entre la nature et l'humanité. Un naturalisme non antihumaniste, autrement dit un naturalisme humaniste est possible dans la mesure où il refuserait le sacrifice de l'humanité au profit de la nature comme Grand Tout.

Le problème, c'est que la position des Larrère est beaucoup plus subversive, et en définitive beaucoup plus antihumaniste - au sens que nous voulons donner à ce terme, et non au sens qu'ils lui accordent qu'ils ne semblent le croire... En effet, l'humanisme consiste très précisément à donner à l'humanité une position privilégiée, et c'est pourtant bien à cette "position privilégiée» qu'ils s'attaquent! Car l'humanisme est d'abord et avant tout une interprétation, au sens nietzschéen du terme, l'évaluation ontologique d'une volonté de puissance. Sans déployer ici tout le mouvement historique qui pourrait en rendre compte, rappelons que le terme d'humanisme ne se trouve pas sous la plume d'Érasme ou de More; on trouve certes celui d'umanista, mais celui-ci désignait alors, comme on le sait, le professeur de grammaire ou de rhétorique. L'humanisme est une construction, une signification

7. Catherine Larrère et Raphaèl Larrère, Du bon usage de la nature, Paris, Aubier, 1997, p. 83-84 louvrage dorénavant noté $D$ ]. 
rétroactive qui empêche de comprendre ce que Cassirer a identifié comme l' «unité systématique» de la philosophie de la Renaissance, une philosophie qui dépasse très largement les limites du «savoir humaniste» : l'humanisme, c'est la Renaissance amputée, c'est le microcosme sans macrocosme, Giordano Bruno à nouveau mis sur le bûcher ${ }^{8}$. Et si l'on tente de définir un humanisme originel, et l'humaniste comme celui qui fait ses humanités, on succombe à cette construction! Répétons-le encore une fois, il n'y a d'humanisme et d'humaniste, au sens où il nous faut désormais entendre ces termes, que construits après-coup (et, d'une certaine manière, construits contre la Renaissance).

Cette construction se déploiera au moment précis où il s'agira d'assurer la possibilité historique d'un développement sans limite de l'humanité: le take off de l'économie capitaliste, le décollage qui a lieu au moment de la dite "révolution industrielle» en Angleterre dans les années 1750-1800, s'est effectué en même temps que le take off humaniste. Ce take off de l'humanisme a consisté à faire de la position d'exception de l'homme la justification de la domination en même temps que la cause de la dévastation du monde - ce que nous nommons l'humanisme globalisé (une expression qui ne fait, en définitive, que participer à cette relecture écologiste, ce "nouveau récit» de la Révolution industrielle qu'analyse François Jarrige dans ce numéro). C'est à partir de cette justification que les soi-disant «humanistes» ont été relus et nommés en tant qu'humanistes, afin de transformer le "privilège " et la «dignité» de l'homme en signe d'exception. Pour Érasme, «l'homme ne naît pas homme, il le devient ». Ce devenir signifie que l'humanité n'est pas donnée, mais doit être cultivée et produite comme telle, arrachée à la nature. Ce qui fait la «dignité» de l'homme pour Pic de la Mirandole, c'est précisément la possibilité toujours offerte à l'homme de se définir sur fond d'un manque de définition: l'homme, "cette œuvre à l'image indistincte (indiscretae opus imaginis)», sans «place déterminée, ni visage propre», doit se «définir» lui-même, et «achè$\mathrm{ve}[\mathrm{r}]$ [s]a propre forme librement, à la façon d'un peintre (plastes) ou

8. Ernst Cassirer, Individu et cosmos dans la philosophie de la Renaissance, Paris, Minuit, 1983, p. 7, 13 et 126. Sur cette « unité » de la philosophie de la Renaissance, qui va bien au-delà d'un quelconque humanisme, voir Ernst Bloch, La Philosophie de la Renaissance, Paris, Payot, 2007, qui montre comment la Renaissance est impensable sans l'essor du capitalisme. 


\section{Écologistes, encore un effort pour devenir antihumanistes...}

d'un sculpteur (fictor $)^{9} »$. En ce sens, lorsque Foucault en 1966 définit I'humanisme comme ce qui « promet l'homme à I'homme ${ }^{10}$ ", il montre le résultat annoncé d'une telle autodéfinition. C'est pour cela que nous accentuons encore plus qu'il ne le fit la formule de Foucault, nous l'entendons au pied de la lettre, comme formule de l'autisme humaniste effectué par le capitalisme. C'est parce que l'humanisme ne vise qu'à la conception - la pensée et la production - de l'homme comme homme vers l'homme, que tout ce qui n'est pas l'homme est dévalorisé.

Mais ce n'est pas seulement un problème axiologique, ou moral, il ne s'agit pas seulement d'attaquer l'excessive mise en valeur de l'homme comme le font trop souvent les écologistes «profonds». Ce qu'il nous faut comprendre, c'est l'articulation de l'humanisme à la production économique capitaliste. La difficulté est la suivante: la modernité place l'homme en position transcendante là où le capitalisme effectue l'immanence du monde, une forme d'immanence saturée que construit le marché global, (la «dépendance universelle», le «marché mondial », le " commerce universel » déjà décrit par Marx et Engels dans L'Idéologie allemande en 1845). Il faut donc complexifier le modèle proposé par les Larrère: avec la modernité capitaliste réalisée, I'homme est à la fois hors du monde et dans le monde, bulle de transcendance circulant dans une immanence liquide. Tout se passe comme si désormais l'exception humaniste se produisait à même les flux de capitaux, d'images et de travailleurs. Le capitalisme a digéré l'humanisme, mais ne l'a pas entièrement liquidé, il l'a métabolisé: quelque chose dans les flux de consommation circule et se maintient comme tel, c'est la "dignité" ontologique de l'homme. En ce sens, le capitalisme est un humanisme emporté vers sa dernière extrémité. Désormais, pour reprendre les termes de Pic de la Mirandole, l'homme est réellement une «œuvre à l'image indistincte», il n'a réellement «ni place déterminée, ni visage propre, ni don particulier», il doit réellement se «définir» lui-même, et «vouloir» et «conquérir par lui-même» sa «place», en bon entrepreneur-de-soi néolibéral. Toutes les définitions négatives mises en avant par Pic-l'homme n'est «ni céleste ni terrestre, ni mortel ni immortel » - qui font de l'être humain un être «souverain de [lui]-même», capable

9. Jean Pic de la Mirandole, « Sur la dignité de l'homme », dans Euvres philosophiques, Paris, PUF, 1993, p. 6-7.

10. Michel Foucault, Dits et Écrits I. Paris, Gallimard, «Quarto », 2001, p. 569. 
d' «[achever sa] propre forme librement», sont aujourd'hui mises à contribution dans le capitalisme postmoderne. L'être humain est flexible. Se forme en permanence. Et constitue son Capital humain. Le capitalisme contemporain promet l'homme-flexible à l'homme, un homme sans essence, comme le dit la tradition métaphysique, c'est-àdire capable de devenir n'importe quoi.

Le capitalisme n'a pas liquidé le privilège de l'homme, ni l'humanisme protégé l'homme du capitalisme, nous avons simplement assisté à des transformations dans la façon de concevoir ce privilège. Ce qu'on peut dire, c'est que le sujet du capitalisme est en phase avec le monde créé à son usage, la ligne des flux de marchandises et de capitaux est entrée en résonance avec celle des flux de productivité et d'auto-productivité humaine; "tout branle». L'exception humaniste s'avère de plus en plus pour ce qu'elle est: une procédure d'indemnisation fallacieuse, d' «auto-immunisation» (Derrida) par laquelle l'être humain tente de maintenir la croyance profonde en son immortalitél!.

\section{L'antihumanisme de l'écologie politique}

On a souvent pu le théoriser, le capitalisme est sans limite, et ne s'écroulera pas sous ses propres contradictions: la seule limite au capitalisme et ses mécanismes de destructions prédatrices, c'est l'endommagement irréversible des possibilités de vie humaines et non humaines. Cet endommagement, l'écologie politique le pointe, jour après jour. Et ne peut donc que se construire sur une analyse critique du capitalisme. Il nous semble que cette critique ne peut prendre toute sa dimension qu'accompagnée d'une critique de l'humanisme, au sens que nous avons tenté de dégager. En un mot, pas d'anticapitalisme véritable sans antihumanisme, si l'on entend bien, encore une fois, qu'il ne s'agit pas dans cet antihumanisme de danser autour d'un feu en souhaitant la disparition de l'espèce humaine...

Là n'est pourtant pas le terme de la pensée écologiste, seulement son point de saillance politique. En effet, pour être sensible à l'atteinte

11. Nous renvoyons sur ce point à notre article $*$ De la civilisation comme crash-test », Ctheory, site http://www.ctheory.net/articles.aspx?id=580, août 2007 [consulté le 15 mai 2008], ainsi qu'à L'Indemne. Heidegger et la destruction du monde, Paris, Sens et Tonka, 2008. 
Écologistes, encore un effort pour devenir antihumanistes...

aux formes de vie, pour interroger la place de l'être humain et le danger de sa mise en exception, il faut aussi être capable de proposer un autre schéma de relations - l'espace des relations écosystémiques dans lequel l'être humain se découvre inséré, le rapport de l'espèce humaine aux autres espèces vivantes. L'antihumanisme propre à toute pensée écologisée ne peut pas s'arrêter à une simple critique radicale de l'humanisme et du capitalisme, il lui faut remettre en cause les Grands Partages qui clivent l'humanité des non-humains: sur ce clivage repose la mise en exception humaniste.

Que serait en effet un antihumanisme achevé? Ni plus ni moins qu'une sortie de l'anthropotechnique du capitalisme. Une remise en cause du paradigme vertical de l'arrachement à la nature par lequel l'être humain se définit comme sans-essence. Cette remise en cause, la pensée écologisée peut y contribuer en proposant un autre schéma de relations, qui envisagerait une forme d'égalité entre les êtres humains et les non-humains, une «démocratie des formes de vie» (Arne Naess), une véritable cosmopolitique. 